\title{
HIGH DISPERSION SPECTRA OF BRIGHT PLANETARY
}

\author{
NEBULAE \\ SIEK HYUNG and LAWRENCE H. ALLER \\ University of California, 8979 Math Science bldg., 405 Hilgard Ave. \\ Los Angeles, California 90024-1562
}

The Hamilton Echelle Spectrograph at the coudé focus of the Shane $3 m$ telescope at Lick Observatory permits us to obtain high resolution spectra of bright planetary nebulae over the spectral range from $3500 \AA$ to $10300 \AA$. Not only is it possible to separate pairs such as $\lambda 5198,5200$ [NI], $4860 \mathrm{HeII}$ and $4861 \mathrm{HI}$, but one may secure the profiles of Doppler broadened lines. It appears to be possible to separate the $\mathrm{HI}$ and HeI contributions of the 3889 line in $\mathrm{Hu}$ 1-2. The nebulae most suitable for observation are those of high surface brightness. NGC 7027 was the first nebula intensively studied with this equipment (Keyes et al. 1990); subsequently we have observed NGC 2440, 6537, 6543, 6567, 6572, 6741, 7009, 7662, IC 351, 418, 2149, 2165, 4634, 4997, 5217, $\mathrm{Hb} 12$, and Hu 1-2. In NGC 7009 we measured line fluxes at the ends of major and minor axes. Small compact objects were centered on the slit; other were observed in the bright ring. These planetary nebulae cover a range in excitation level, chemical composition, and evolutionary status.

A comparison of Hamilton Echelle data with those obtained with an image tube scanner reveals that echelle data are often an order of magnitude more accurate. Part of this superiority arises from the high spectral resolution of the echelle, which not only enables one to separate lines in blends but also permits the position of the continuum to be estimated more confidently; other benefits arise from from the linearity of the CCD detector. High spectral resolution can increase the number of lines for plasma diagnostic uses and it yields data on internal motions as noted above. Improved quality and quantity of nebular line measurements are essential to assess theoretical $A$-values and collision strengths since no laboratorial checks are possible. The high spectral resolution permits as to separate lines of stellar and nebular origin. Stellar lines are often diffuse and their profiles invariably differ from the Doppler broadened nebular emissions.

An objective of this investigation is to provide data for improved abundance determinations and theoretical nebular models. For a number of these nebulae such as NGC 7009 and IC 4997 we observed many permitted lines of CII, NII, OII, SiII etc. that are excited by recombination and cascade. Iron is observed in various ionization stages in several objects. Some of the weaker lines may arise from shock excitation and assist us in the construction improved sophisticated nebular models in which evolutionary effects are taken into account. 